\title{
Nonlinear dynamics of confined liquid systems with interfaces subject to forced vibrations
}

\author{
María Higuera, Jeff Porter, Fernando Varas, José M. Vega *
}

\section{Contents}

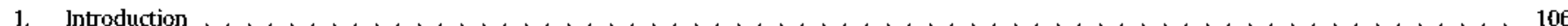

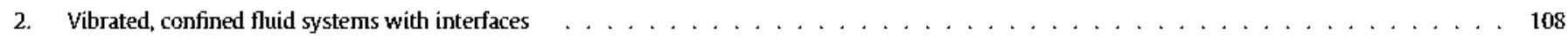

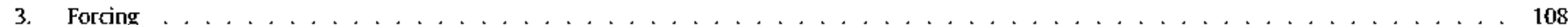

3.1. Direct forcing in vibrated containers $\ldots \ldots \ldots \ldots \ldots$

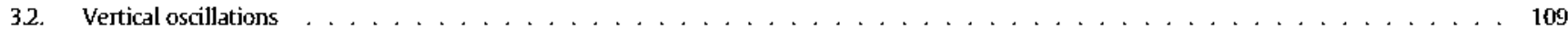

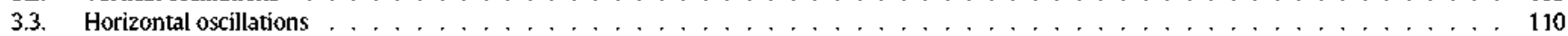

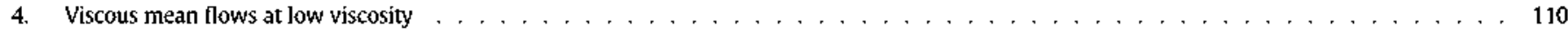

4.1. Interaction of the viscous mean flow and nearly inviscid Faraday waves in almost circular containers . . . . . . . . . . . . 111

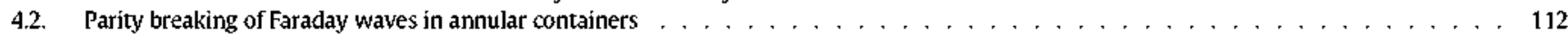

5. A preliminary analysis of compressed-expanded monolayers over a liquid layer $\ldots \ldots \ldots$

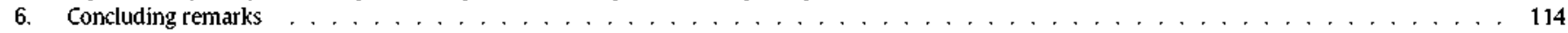

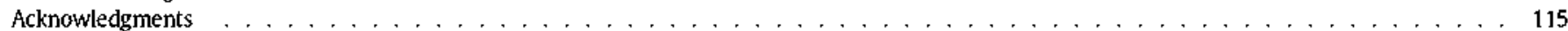

References . . . . . . . . . . . . . . . . . . . . . . . . . . . . . . . . . . . . . . . . .

\section{Introduction}

Isolated, static (steady) systems are an idealization that rarely holds up under strict scrutiny. Parameters that are assumed to be constant in the mathematical formulation (the gravitational acceleration, for example) can drift slowly in time, exhibit rapid oscillations, or both. Rapid fluctuations are unavoidable due to ambient noise and perturbations whose magnitude may be significant in some cases. For instance, crew maneuvering and on-board machinery produce time dependent mechanical forcing ( $g$-jitter [1]) in space laboratories that persists in the absence of an effective damping mechanism. Time dependent perturbations introduce dynamics into the system that may or may not significantly change the system state, depending on the associated timescales and the sensitivity of the system. For instance, g-jitter may produce significant effects on on-board material processing and experiments [2] involving confined fluid systems with interfaces, which are very sensitive to time dependent mechanical forcing because, except at very small size (the scope of microfluidics) and very large vibrating frequencies, ordinary liquids exhibit very small viscous damping. The 
effects of vibrations on fluids are important in a wide range of other scientific and engineering applications such as liquid storage, mixing, convection, pattern formation, and the study of basic fluid instabilities.

Ambient vibrations are generally broad band and exhibit time dependent amplitude and direction. However, these oscillations are transmitted to the fluid system through the natural modes of the support structure and the container, meaning that the actual forcing experienced by the fluid usually peaks at some well defined frequencies, amplitude, and directions. Hence, the case of monochromatic oscillations, with fixed amplitude and direction, is a good idealization that provides considerable insight into the relevant physical mechanisms and the subsequent response of the fluid system.

In many important situations, the response of the system occurs on a lengthscale that is small compared to the extent of the system. Distinct excitation patterns may then compete with one another, leading to complex dynamical behavior and pattern selection phenomena. Extended pattern forming systems of this type occur throughout physics, chemistry, and biology, as well as fluid dynamics, and much effort has gone to understanding the factors that determine the nature of the selected states: regular or irregular, steady or periodic, delicate or robust under perturbations, etc. An important distinction can be made between those aspects of the pattern that depend on detailed physical properties, and those which result more generally from its symmetries. Also, due to the different phenomena involved, the effects of vertical and horizontal vibrations are considered separately.

The paradigmatic example of vertical periodic forcing is the Faraday system [3], in which a container of fluid is shaken in periodic fashion to provoke surface waves. Although Faraday himself explored both vertical and horizontal shaking, subsequent attention has overwhelmingly favored the vertical case, now recognized as a classic example of parametric instability [4]. The Faraday system is relatively compact, evolves on a convenient time scale, and is conducive to simple controlled experiments. It also yields a tremendous variety of patterns [5-8], depending on the applied forcing function and fluid properties. The theoretical analysis of Faraday waves can be greatly simplified by assuming a perfectly flat horizontal surface undergoing perfectly vertical vibrations. The forcing is uniform and, in a comoving frame, acts simply as an amplitude modulation of the gravitational force, a purely parametric forcing mechanism. The flat surface solution persists in the presence of this forcing despite its eventual loss of stability at critical forcing amplitude. Despite their central role in the analytical treatment of the vertically forced Faraday problem [9], the twin assumptions of a flat horizontal surface subjected to purely vertical shaking are, in many situations, restrictive. Both assumptions will fail, at some level, for any realistic experiment due to the influence of surface tension, which dominates at small scales and under microgravity conditions, and produces capillary (or meniscus) waves near the boundaries. Although these can be suppressed through careful control of boundary conditions [5,10], such synchronous, spatially nonuniform disturbances are a generic feature of vibrated fluids in most configurations. This observation can be used to argue that, although more resistant to theoretical analysis, horizontally or obliquely vibrated systems may be more relevant to the question of general fluid behavior than the more popular vertically forced Faraday system.

Horizontal forcing leads to a range of interesting new phenomena, some of which were noted by Faraday [3], who observed 'a series of apparently permanent ridges projecting outward like the teeth of a coarse comb' from the edge of a submerged vibrating plate [11]. These ridges can be identified with the subharmonic waves known as crosswaves, which are produced by a wavemaker (namely, a horizontally vibrating plate or similar device used to excite traveling waves on the surface) in a semi-infinite container; see [11] and references therein. Cross-waves are observed in a variety of wavemaker experiments [12], and can display complex behavior, including chaotic dynamics and slow modulations resembling solitary waves [13]. Not only is the cross-wave instability dramatic (quickly overwhelming the underlying synchronous waves in many cases) and easily observed across a broad range of fluid configurations and forcing, but it arises from a parametric instability [14] at the boundary (wavemaker), making it the localized counterpart of the usual Faraday wave instability that has produced so many intriguing patterns. Horizontal vibrations in rectangular containers that are only moderately large allow for interaction between the vibrating endwalls that, unlike standard cross-waves in larger containers, produces patterns that are neither perpendicular to the vibrating end-walls nor strictly 2:1 subharmonic. Instead, these subharmonic waves are oblique, as recently observed experimentally [15] and confirmed theoretically with good agreement in [16], where the dynamics have been shown to be quasiperiodic; some of the results in [16] will be anticipated in the present review. It is to be noted that, unlike the Faraday system, former analyses of horizontally vibrated containers concentrated either on small containers [17-20] (in which the instability only involves a few modes) or fully nonlinear behavior, using modal expansions [21-23] and finite differences [24], which provide simulations but do not allow for extracting more useful knowledge about the associated dynamics. A deeper analysis, similar to the one already performed for the Faraday system is lacking in the literature.

Vibrations always induce viscous mean flows, which had been seen as a byproduct of the oscillating flow in early studies but were more recently shown by some of the authors to be coupled with the primary vibrating field [25]. This leads to new attractors and dynamical phenomena that are due to the interaction with the mean flow. Viscous mean flows rely on localized viscous effects but, surprisingly enough, they produce an overall circulation in the bulk fluid that does not disappear as viscosity goes to zero [26]. Also, these flows are slowly varying (compared to the primary oscillating field) at low viscosity, which facilitates interaction with slow interfacial phenomena, such as Marangoni elasticity [27].

Some experimental devices [28] used to measure non-steady rheological properties involve (very slow) oscillations of surfactant monolayers over a fluid promoted by horizontal oscillations of two parallel barriers, which move in counterphase in a symmetric way. The barriers are only slightly immersed in the fluid, with the intent of minimizing the fluid dynamics of the bulk phase. The question of whether the fluid dynamics can legitimately be ignored has not been addressed in the literature to our knowledge; a preliminary analysis will be provided at the end of the paper. On the other hand, the oscillations are so slow that coupling to capillarity-gravity waves produced by external forcing is unlikely. The question remains whether coupling with fluid dynamics is possible due to slower dynamical effects and mean flow is a good candidate to enhance such coupling. Furthermore, the experimental devices usually possess symmetries that can affect the expected dynamics. As an alternative to current experimental devices, rheological properties can also be measured using much faster mechanical vibrations combined with a good theoretical model that simulates the system [29]. This, however, requires a good understanding of the fundamentals of the dynamics of vibrating fluid systems with interfaces, which is the purpose of the present paper. Specifically, this review explains in simple terms the differences between direct and parametric forcing, as well as the different behaviors that are to be expected from vertical and horizontal excitations. The review concentrates on the small viscosity limit, which is the relevant one for ordinary liquids, and focusses on the effect of symmetries and nonlinearity in the response of the system. In addition, the role of viscous mean flows is illustrated, showing that it couples with the primary surface waves and promotes coupling of the fluid dynamics with surface viscosity, which fundamentally affect the system response. Coupling of the fluid dynamics with surface rheology is further emphasized with a preliminary analysis of the dynamics of compressed-expanded monolayers over a liquid layer.

With this general context in mind, we continue with the formulation of the problem in terms of the Navier-Stokes equations (Section 2) and then consider the forcing mechanisms (Section 3), the viscous mean 
flow (Section 4), and the above mentioned compressed-expanded monolayers (Section 5). Concluding remarks are given in Section 6.

\section{Vibrated, confined fluid systems with interfaces}

Because of viscous dissipation (which is present in all realistic fluid systems), any initial perturbation will eventually be damped out and the system will either settle back towards the original unperturbed state or drift to an alternative equilibrium favored by the initial conditions. To maintain dynamics over time, the forcing must be maintained as well. A balance between forcing (the source of energy) and dissipation (which acts as a sink of mechanical energy) must be achieved. Below, for concreteness, we consider a horizontal container vibrated in a rigid-solid fashion, although the ideas apply to more general vibrated systems with interfaces, such as vibrating drops [30-32] and surface waves generated by immersed wavemakers [33]. The starting point to describe the vibrated liquid system is the incompressible Navier-Stokes equations, which are written in a moving Cartesian reference frame attached to the container, with the $x$ and $y$ axes at the unperturbed free surface, assumed to be perfectly flat and horizontal, and the $z$ axis pointing upwards. With the usual notation $(t=$ time, $\mathbf{v}=$ velocity, $p=$ stagnation, hydrostatic pressure, $\nabla=$ gradient operator, $\rho=$ density, and $\nu=$ kinematic viscosity), the governing equations in the moving frame are

$\boldsymbol{\nabla} \cdot \boldsymbol{v}=0, \quad \partial_{t} \boldsymbol{v}-\boldsymbol{v} \times \boldsymbol{\nabla} \times \boldsymbol{v}=-\nabla p / \rho+\nu \nabla^{2} \boldsymbol{v}$.

The equations apply in the bulk fluid, ( $x, y) \in \Omega_{S},-d<z<f$, where $\Omega_{S}$ is the cross section of the container, $d$ is the container depth, and $f$ is the free surface elevation. The boundary conditions at the free surface $z=f$,

$\left(\partial_{t} f\right)\left(\boldsymbol{e}_{z} \cdot \boldsymbol{n}\right)=\boldsymbol{v} \cdot \boldsymbol{n}, \quad\left[\left(\boldsymbol{\nabla} \boldsymbol{v}+\nabla \boldsymbol{v}^{\top}\right) \cdot \boldsymbol{n}\right] \times \boldsymbol{n}=0$,

$$
\begin{aligned}
& p-\rho|\boldsymbol{v}|^{2} / 2+\rho \boldsymbol{a} \cdot\left(x \boldsymbol{e}_{x}+y \boldsymbol{e}_{y}+f \boldsymbol{e}_{z}\right) \omega^{2} \cos \omega t+\rho g f \\
& +\sigma \nabla \cdot\left[\boldsymbol{\nabla} f /\left[\sqrt{1+|\boldsymbol{\nabla} f|^{2}}\right]=\rho v\left[\left(\boldsymbol{\nabla} \boldsymbol{v}+\boldsymbol{\nabla} \boldsymbol{v}^{\top}\right) \cdot \boldsymbol{n}\right] \cdot \boldsymbol{n},\right.
\end{aligned}
$$

account for kinematic compatibility and equilibrium of tangential and normal stresses, respectively, Here, $\mathbf{a}$ is the forcing amplitude, $\omega$ is the forcing frequency, $\boldsymbol{e}_{x}, \boldsymbol{e}_{y}$, and $\boldsymbol{e}_{z}$ are the unit vectors along the $x, y$, and $z$ axes, respectively, $g$ is the gravitational acceleration, $\sigma$ is the surface tension, $\boldsymbol{n}$ is the upward unit normal to the free surface, and $\boldsymbol{\nabla} \boldsymbol{v}^{\top}$ is the transpose of the strain rate tensor $\nabla \boldsymbol{v}$. No-slip is imposed at the solid walls, namely

$\boldsymbol{v}=\boldsymbol{O}$ at $(x, y) \in \boldsymbol{\Gamma}_{S}$ and at $z=-d$,

where $\Gamma_{S}$ is the boundary of $\Omega_{S}$ (which defines the lateral walls).

This formulation is not complete if $\sigma \neq 0$ because of the second order spatial derivatives of $f$ appearing in the boundary condition (3). Fixing the contact line, namely imposing that

$f=0 \quad$ at $(x, y) \in \mathbf{\Gamma}_{S}$,

(which can be achieved experimentally using either sharp edges or hydrophobic coatings) is a good strategy to avoid uncertainties associated with contact line motion, whose modeling is still not well understood [34]. These uncertainties can mask comparison of theory with experiments and make it hard to distinguish an appropriate theory from an incorrect one.

We assume here that the surface tension is constant. Thermal gradients and/or surface contamination would make $\sigma$ dependent on the temperature and surfactant(s) concentration, and require (i) adding new terms in the second boundary condition (2) that account for tangential stresses proportional to the surface tension gradients, as well as terms accounting for shear and dilatational surface viscosity, and (ii) considering new conservation equations to calculate the instantaneous temperature and surfactant concentration fields.

Viscous effects are usually weak. Specifically, the ratio of inertia $\left(\partial_{t} v\right)$ to viscous $\left(\nu \nabla^{2} v\right)$ terms in Eq. (1), which is measured by the StrouhalReynolds number,

$S t R e=\omega \ell^{2} / \nu \gg 1$,

where $\ell$ is the wavelength of the excited waves, is large. ${ }^{1}$

For ordinary liquids, $v$ is quite small (of the order of $0.01 \mathrm{~cm}^{2} / \mathrm{s}$ ), which means that assumption (6) holds except for very large $\omega$ or very small $\ell$. For instance, if $\omega \sim 10 \mathrm{~Hz}$, then assumption (6) holds provided that $\ell \gg 1 / \sqrt{\nu / \omega}=1 / \sqrt{0.001 / \pi}=0.013 \mathrm{~cm}$. The wavelength $\ell$ is of the order of the size of the system for both nonresonant oscillations and resonant oscillations associated with the lowest natural frequencies (known as sloshing modes). For resonant oscillations associated with higher order modes, $\ell$, can be much smaller than the size of the system, namely of the order of $2 \pi / k$, where $k$ is the gravity-capillary wavenumber given by the dispersion relation $\omega^{2}=g k+\sigma k^{3} / \rho$. At small damping, the fluid system is nearly conservative and can be seen as a collection of (infinitely many) weakly damped harmonic oscillators.

In addition, we assume that the steepness of the surface waves is small, which requires

$|a|,|f| \ll \ell$.

\section{Forcing}

It is customary to distinguish two types of external forcing mechanisms, namely, direct and parametric forcing. Direct forcing is best understood as a driving that immediately changes the system, breaking the static equilibrium already for infinitesimal forcing amplitude. Parametric forcing is so-called because, in its simplest form, it can be understood as a modulation of a parameter of the system. For instance, in a harmonic, linear oscillator (modeling, e.g., small oscillations of a simple pendulum around the stable equilibrium), direct and parametric forcing are modeled as

$\ddot{u}+\gamma \dot{u}+\omega_{0}^{2} u=a \cos \omega t$,

$\ddot{u}+\gamma \dot{u}+\left(\omega_{0}^{2}+a \cos \omega t\right) u=0$,

respectively. In the pendulum, these correspond to horizontal (Eq. (8)) and vertical (Eq. (9)) oscillations of the support. The essential difference between these is that with direct forcing $u \neq 0$ if $a \neq 0$, while the state $u=0$ persists with the parametric forcing of Eq. (9) (named after Mathieu [35]) even if $a \neq 0$. In the parametric case, nonsteady dynamics appear as the state $u=0$ loses' stability, which, using Floquet theory, requires that $a$ exceeds a threshold value $a_{\mathrm{c}}$. The instability region in the $\omega-a$ plane is defined by a series of cusped resonance tongues that approach $a=0$ at discrete values of $\omega / \omega_{0}$ as $\gamma \rightarrow 0$. The most dangerous of these (requiring the smallest forcing amplitude with finite damping) is centered at $\omega / \omega_{0}=2$ (i.e., the forcing frequency is twice the natural frequency) and is called the 2:1 parametric resonance because the unstable mode is periodic, with a frequency approximately equal to one half of the forcing frequency. This parametric instability can be understood noting that if $u \sim \cos \omega_{0} t$ is resonant and $\omega=2 \omega_{0}$, then the product of $\cos \omega t$ and $u$ produces a resonant term proportional to $\cos \left(2 \omega_{0}-\omega_{0}\right) t=\cos \omega_{0}$ t. The same argument

\footnotetext{
' Sometimes, this nondimensional number is misleadingly called a 'Reynolds number', but it is the product of the Strouhal $(\omega \ell /|\boldsymbol{v}|)$ and the Reynolds $(|\boldsymbol{v}| \ell / \nu)$ numbers, and is independent of the velocity, which is an essential difference with the Reynolds number.
} 
shows that, if nonlinear terms are added to Eq. (8), then the harmonic oscillations also produce parametric forcing through quadratic terms, which act similarly to those resulting from the product of $\cos \omega t$ and $u$ in Eq. (9). If quadratic terms are not present then subharmonic oscillations are not triggered. This occurs, in particular, if the nonlinear extension of Eq. (8) is invariant under the action

$u \rightarrow-u, \quad t \rightarrow t+\pi / \omega$.

This symmetry is present in the simple pendulum, the nonlinear equation for which is obtained by substituting $\sin u$ for $u$ in the final terms on the left-hand-sides of Eqs. (8) and (9).

In this manner, the symmetries of the equation can be used to anticipate some properties of the dynamics. In particular, the difference between direct and parametric forcing is fundamental and related to the distinction between forced and spontaneous symmetry-breaking. Eq. (9) is invariant under the reflection symmetry $u \rightarrow-u$, which implies the equivalency of the solutions $\pm u(t)$ and the existence of the equilibrium $u(\mathrm{t})=0$. The forced symmetry-breaking introduced by the driving term in Eq. (8) immediately breaks the reflection symmetry and prohibits the steady solution $u(t)=0$. The system is forced toward a dynamical state (lacking reflection symmetry) for any finite forcing $a$. This does not occur in Eq. (9), where the reflection symmetry $u \rightarrow-u$ remains intact and the steady solution $u(\mathrm{t})=0$ remains stable for small $a$. An instability (symmetry-breaking bifurcation) occurs at a critical value of $a$ and only after this point will the system respond in a lasting way to small perturbations. This distinction between continuous response (direct forcing) and threshold response (parametric forcing) is fundamental. Direct forcing mechanisms have an immediate effect on the state of the system while parametric forcing mechanisms affect the initial state indirectly, allowing it to persist but (potentially) altering its stability for sufficiently large forcing values.

In some cases, vibrations have a stabilizing effect. A classical example [36], first uncovered by Stephenson [37], is the (unstable) upper equilibrium of a pendulum subject to vertical vibrations of the support (still given by the Mathieu equation, but with the sign of $\omega_{0}^{2}$ reversed). This equilibrium is stabilized if $\omega \gg 1$ and the vibrating acceleration is in the appropriate range.

Both the direct and parametric forcing mechanisms discussed above are present in vibrated containers, as seen in the following subsections.

\subsection{Direct forcing in vibrated containers}

Direct forcing produces harmonic oscillations whose frequency is close to the forcing frequency. This occurs in generic conditions because the boundary condition (3) implies that the quiescent state $\boldsymbol{v}=\mathbf{0}$, $p=0, f=0$ is not generally a solution when $\boldsymbol{a} \neq \mathbf{0}$. Direct forcing can be either resonant or nonresonant, depending on the forcing frequency (and the symmetries of the problem, as discussed below). Both resonant and nonresonant oscillations can be understood considering the linearization of Eqs. (1)-(5) around the quiescent state, neglecting viscous effects (which are assumed to be small, according to Eq. (6)). If, in addition, the velocity is eliminated using the inviscid, linear approximation of Eq. (1), namely

$\boldsymbol{\nabla} \cdot \boldsymbol{v}=0, \quad \partial_{t} \boldsymbol{v}=-\nabla p / \rho$,

then $p$ and $f$ are determined by the following inviscid linear problem

$$
\begin{aligned}
& \nabla^{2} p=0 \quad \text { in }(x, y) \in \Omega_{S},-d<z<0, \\
& \rho \partial_{t t} f+\partial_{z} p=0, \\
& \quad p+\rho g f+\sigma \nabla^{2} f+\rho \omega^{2} \boldsymbol{a} \cdot\left(x \boldsymbol{e}_{x}+y \boldsymbol{e}_{y}\right) \cos \omega t=0 \text { at } z=0,
\end{aligned}
$$

$\boldsymbol{\nabla} p \cdot \boldsymbol{n}_{S}=0, f=0$ at $(x, y) \in \Gamma_{S}$, $\partial_{2} p=0$ at $z=-d$,

where $\boldsymbol{n}_{S}$ is the outward unit normal to $\Gamma_{S}$.

Note that neglecting viscous effects only permits one to impose that the solid walls and free surface are impenetrable, but does not allow one to fully impose the no-slip boundary condition (4) or the second boundary condition (2) at the free surface. These are imposed by considering two oscillatory boundary layers $[38,39]$ near the free surface and the solid walls, where viscous effects cannot be neglected. These boundary layers play an essential role in both the calculation of viscous damping and the generation of the viscous mean flow, as discussed below.

For generic values of $\omega$ and the direction of a, the linear problem (12) -(15) exhibits a unique solution, which defines non-resonant oscillations. At certain specific values of $\omega$ (the natural frequencies), in contrast, this problem does not generally have a solution. The natural frequencies are defined such that the homogeneous version of Eqs. (12)-(15) (setting the forcing amplitude a to zero) possesses nontrivial solutions, which are the natural modes. The amplitude of resonant oscillations cannot be determined by the inviscid problem, but requires including viscous effects in Eqs. (12)-(15), which can be done, for instance, using a quasipotential approximation [40]. It should be noted that treating viscous damping is rather subtle due to the fact that it is produced by two distinct effects: viscous dissipation in the boundary layers and in the bulk. The strength of these depends on the dimensions of the container and the capillary-gravity balance [41].

This is the general picture, but symmetries (of either the forcing or the container) may have important qualitative effects, suppressing some of the resonances expected in the generic case. As with the simple pendulum, this linear problem illustrates clearly the different nature of vertical and horizontal oscillations.

\subsection{Vertical oscillations}

For vertical oscillations, $\boldsymbol{a}=\boldsymbol{a} \boldsymbol{e}_{2}$, the forcing term in the boundary condition (13) vanishes and there is no direct forcing. Parametric forcing, instead, is analyzed by retaining the term proportional to

$\rho\left(\boldsymbol{a} \cdot \boldsymbol{e}_{2}\right) \omega^{2} f \cos \omega t \equiv \rho a \omega^{2} f \cos \omega t$

(see Eq. (3)) in the boundary condition (13) and including the effect of viscous damping. That term was omitted in Eq. (13) because, since $|\boldsymbol{a}|$ and $|f|$ are both small compared to $\ell$ (see Eq. (7), where $\ell$ is not larger than the size of the container), this term is small compared to the direct forcing term. As with the simple pendulum, the quiescent solution persists and oscillations appear only as a result of parametric instability. The simplest such oscillations are 2:1 subharmonic and are produced by the forcing term (16) through the same mechanism discussed in connection with Eq. (9). In addition, this system produces quasiperiodic oscillations involving two natural modes, which were not possible in Eq. (9) because it only contains one natural mode. The strongest of these are quadratic, and occur when

$\omega_{1} \pm \omega_{2} \simeq \omega$

where $\omega_{1}$ and $\omega_{2}$ are two natural frequencies. As in the 2:1 resonance ( $w h i c h$ is a particular case of Eq. (17), for $\omega_{1}=\omega_{2}=\omega / 2$ ), the instability occurs because the product of each natural mode with $\cos \omega t$ yields a term with a frequency close to the other natural mode. This is just because of the trigonometric relation giving $\cos \omega t \cos \omega_{1} t=\left[\cos \omega_{2} t+\right.$ $\left.\cos \left(2 \omega_{1} \pm \omega_{2}\right) t\right] / 2$ (and its counterpart interchanging $\omega_{1}$ and $\omega_{2}$ ).

Surface wave patterns at threshold are standing and are readily calculated for low forcing frequencies (i.e., comparable to the first natural mode). For higher forcing frequencies, the threshold patterns are typically regular rolls, squares, triangles, or hexagons $[6,40]$ that fill the whole container, but (in spite of the long history of attention to vertical vibrations) the theoretical determination of the patterns and their 
dynamics is still an open problem in three dimensions. The dynamics beyond threshold, at larger forcing amplitudes, involve nonlinear effects that promote increasingly complex behavior [6], including propagative patterns, which are enhanced by the mean flow (Section 4). As in the linear response discussed above, an important question with forced fluid systems, and dynamical systems in general, is the extent to which symmetries, either exact or approximate, constrain the observed behavior. This is evident in multi-frequency forced Faraday waves $[8,42]$. If the surface waves are weakly damped, the broken symmetries of time translation, time reversal, and Hamiltonian structure can be exploited $[43,44]$ to obtain general scaling laws and parameterdependence for the coefficients of the amplitude equations that govern their behavior near onset. Despite relying on imperfect (broken) symmetries, such constraints have significant implications, determining which values of the forcing frequencies and their associated phases could be used to enhance or suppress specific resonant interactions, thereby controlling the pattern formation process at the nonlinear level. It should be noted that not all symmetries remain influential when (weakly) broken. Some perturbations are singular and immediately induce large changes (loss of stability, chaos [45], bursting [46], etc.) that cannot be anticipated from an analysis of the unperturbed system.

In analogy to the pendulum example, vertical oscillations of an appropriate high frequency may suppress the Rayleigh-Taylor instability [47-49] in an upside down container.

\subsection{Horizontal oscillations}

As mentioned in the Introduction section, horizontal oscillations have received substantially less attention than vertical oscillations and, indeed, the two cases are quite different. Among other reasons, both resonant and non-resonant oscillations are produced in the horizontal case, and solutions are affected by symmetries in a more fundamental way. In particular, the term proportional to $f \cos \omega t$ is absent in the boundary condition ( 3 ) and parametric forcing is produced by quadratic terms (e.g., that proportional to $|\mathbf{v}|^{2}$ in the boundary condition (3)). Terms proportional to the product of cos $\omega t$ (resulting from the harmonic flow generated by direct forcing) and one of the flow variables result in parametric excitation whenever two natural frequencies satisfy the resonance condition (17). As with vertical oscillations, the resulting patterns can be either 2:1 subharmonic, if $\omega_{1}=\omega_{2}$, or quasiperiodic otherwise. This is true for generic containers lacking symmetry. For containers that are reflection-symmetric about a vertical plane (say, the $x=0$ plane) and with forcing perpendicular to the symmetry plane, the product $\boldsymbol{a} \cdot \boldsymbol{e}_{y}=0$ and thus Eqs. (1)-(5) and (12)-(15) are invariant under the spatio-temporal symmetry

$x \rightarrow-x, \quad \boldsymbol{v} \cdot \boldsymbol{e}_{x} \rightarrow-\boldsymbol{v} \cdot \boldsymbol{e}_{x}, \quad t \rightarrow t+\pi / \omega$,

which prohibits 2:1 parametrically excited motions. This is a conjecture that is consistent with some recent results for rectangular geometry [16] and also with the absence of 2:1 motions in the horizontally excited pendulum because of the symmetry (10); the (unpublished) proof is more elaborate here since the symmetry (18) does not prevent the existence of quadratic terms. For instance, if a rectangular container is vibrated perpendicularly to two of its walls, parametrically excited motions exhibit two different frequencies, $\omega_{1}$ and $\omega_{2}$, satisfying Eq. (17). But as the distance between the vibrating endwalls goes to infinity, the flow near each endwall converges to the 2:1 subharmonic cross-waves that are triggered by the vibrating endwall (which acts as a wavemaker) in a semi-infinite rectangular container. This means that the quasiperiodic motions in the rectangular container must be such that the involved frequencies converge to the subharmonic frequency $\omega / 2$ as the distance between the endwalls goes to infinity. In other words, the quasiperiodicity is due to interaction between the vibrating endwalls. These facts have been checked by three of the authors in [16], where a consistent linear theory has been constructed for vibrating rectangular containers whose dimension is large compared to the capillary-gravity wavelength. The theory provides results that compare quite well with previous experimental measurements and visualizations [15]. This good agreement is illustrated in Fig. 1, where the calculated and measured instability thresholds are compared. Weakly nonlinear effects in the limit of high frequency have only been consistently included in the restricted twodimensional case [50].

\section{Viscous mean flows at low viscosity}

Because of quadratic nonlinearity, small amplitude vibrations of physical systems generically produce mean fields that evolve on a slower timescale. These mean fields can either be a simple byproduct of the nonlinear vibration or couple to the primary oscillating field, affecting the dynamics of the system. The quadratic nonlinearity that forces the mean flow is the convective term

$-\boldsymbol{v} \times \nabla \times \boldsymbol{v}$

in the momentum equation in Eq. (1). At low viscosity, the primary oscillating flow satisfies the linear momentum equation in Eq. (11), which means that this flow is potential and satisfies $\boldsymbol{\nabla} \times \boldsymbol{v}=\mathbf{0}$. Thus it does not provide any contribution to the forcing term (19). Nevertheless, the approximation (11) applies only in the bulk, outside the oscillatory boundary layers (already mentioned in Section 3.1) attached to the solid walls and the free surface. In other words, it is precisely in these boundary layers where the mean flow is produced by the quadratic term (19). Crucially, it turns out that the boundary layer mean flow does not converge to zero at the outer edge of the boundary layers, but provides a nonzero velocity and a nonzero stress at the edges of the boundary layers near the solid walls and the free surface. And it is these finite boundary values that induce the viscous mean flow in the bulk. These new forcing terms are independent of the viscosity and quadratic in the amplitude of the primary waves, indicating that the effects of the mean flow cannot be neglected, even in the limit of vanishing viscosity, a striking result considering that these terms are entirely due to viscous effects.

The first theoretical account of the viscous mean flow is due to Lord Rayleigh [51] in his explanation of the observation by Faraday [3] of an anomalous sand accumulation at the bottom of vibrated containers. This effect was similar to the nonuniform dust accumulation (Kundt figures) at the walls of sound tubes (Kundt tubes, [52,39]). This nonuniform sand or dust accumulation is not possible in a purely oscillatory flow, but must be due to a large scale, steady flow, unknown at that time. Rayleigh [51] performed a careful analysis of the oscillatory boundary layer [39] and also observed that the horizontal viscous mean flow velocity converges to a nonzero value at the edge of the boundary layer, but did not pay very much attention to this asymptotic velocity. Rayleigh recognized instead that this effect was related to the formation of steady

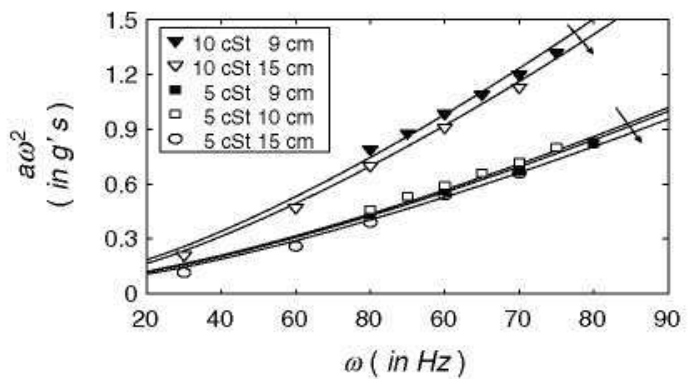

Fig. 1. The dimensional threshold acceleration $a \omega^{2}$ vs. the forcing frequency $\omega$ resulting from for several experimental runs (symbols) and from the theory developed in [16] for $\mu=5 \mathrm{cSt}$ (lower curves) and $10 \mathrm{cSt}$ (upper curves) in a square container whose cross section is indicated; arrows indicate increasing cross section (courtesy of J.M. Perez-Gracia). 
vortical structures in vibrated soap films, already observed by Sedley Taylor [53]. This insight was correct because the vortical structures [54] are generated (at least, if the film thickness does not exceed $1 \mu \mathrm{m}$ ) by the viscous mean flow in the air surrounding the film [55]. The nonzero steady velocity at the edge of the boundary layer was used by Schlichting [38] as a nonzero boundary condition to generate a nonzero circulation in the bulk fluid (precisely, the viscous mean flow). Later on, Longuet-Higgins [56] performed a similar analysis for the oscillatory boundary layer attached to an oscillating free surface. More recently, the viscous mean flow (also called streaming flow or acoustic streaming [57]) has been studied in connection with flows in blood vessels [58], generation of mean motions in the ear [59], interaction of sound waves with obstacles [60] as well as flows around vibrating bodies [61]. In addition, they are of interest in water wave theory (see [62-66] and references therein), play a fundamental role in the generation of Langmuir circulations in the ocean $[67,68]$, and have been studied in connection with standing capillary waves $[69,70]$. Also, concerning material processing in microgravity, the viscous mean flow can be used for controlling undesirable thermocapillary convection [71-73], which promotes striations and other defects in the processed material [74,75].

In all these cases, the primary oscillating flow was given a priori and the viscous mean flow was treated as a byproduct. This is not a correct description in oscillatory fluid flows with interfaces when the primary flow satisfies the generic conditions of being resonant and not strictly standing (see [76] and references therein). In this case, the viscous mean flow affects the dynamics of the primary oscillating flow (i.e., the boundary layers are no longer passive, but become dynamic), a somewhat surprising conclusion to some extent, since the bulk flow remains largely inviscid. The coupling can be explained by noting that the large circulation associated with the viscous mean flow advects vorticity from the boundary layers into the bulk and thus changes in an essential way the initially Hamiltonian nature of the purely oscillatory flow. Furthermore, the mean flow depends quadratically on the amplitudes of the primary waves, while coupling back to the primary waves is via a product of the mean flow and the waves themselves, meaning that, in the end, the coupling term generated by the mean flow is cubic in the amplitude of the vibrating flow, which is of the same order as the typical cubic nonlinearities that are retained in weakly nonlinear descriptions of the primary waves. In such cases the flow in the bulk differs from the flow that would be obtained by ignoring the boundary layers altogether.

In spite of the simple arguments used above to physically justify both the generation of the viscous mean flow and its coupling with the primary waves, the correct formulation of the resulting coupled evolution is a subtle matter that must be handled with care. This is because:

- The mean flow obeys the full Navier-Stokes equations, with (i) some additional terms in the equations accounting for the Stokes drift [52], and (ii) boundary conditions that come from the above mentioned forcing effects near the solid walls and the free surface. The mean flow Reynolds number, Re, which provides the ratio of convective to viscous terms in these equations, scales as

$\operatorname{Re} \sim \omega|A|^{2} / \nu$,

where $A$ is the amplitude of the primary waves and $v$ is the kinematic viscosity. Thus, as $|A|$ increases, Re varies from small to large values, meaning that the dynamics of the mean flow can be quite complex.

- The nature of the mean flow and its coupling to the primary waves depend in an essential way on the symmetries of the system and also on the nature of the primary waves. In particular, solid walls always produce a non-zero mean flow forcing, but the free surface does not produce any forcing if the primary waves are standing (which occurs near threshold in Faraday waves).

- The description contains the full dynamics of the primary waves plus additional terms accounting for coupling to the mean flow. Thus, a full and correct formulation is not yet possible in those cases in which the weakly nonlinear dynamics of the primary waves themselves remains an open problem.

To this point, only the case of Faraday waves with moderate forcing frequency (comparable to the first natural mode) has been formulated in full generality [76]. The case of much larger forcing frequency (which produces pairs of modulated waves) has not been formulated with full generality in three spatial dimensions, among other reasons, because its weakly nonlinear description, even if the mean flow is ignored, is still an open problem. Instead, a phenomenological description [77] was proposed by adding the mean flow to the existing quasipotential approximation for the primary waves [40]. A rigorous formulation of the interaction of modulated waves and the associated mean flow has been derived from first principles only in the twodimensional case, both for vertically [25] and horizontally [50] containers. In the former case, the resulting set of coupled amplitude mean flow equations has been used to show that the mean flow changes in an essential way the dynamics of the primary waves [78]; see [26] for further references.

A full formulation of these systems is well outside the scope of this review. Instead, two particular cases of Faraday waves will be considered that illustrate the ability of the mean flow to affect the dynamics of the primary waves, the role of the symmetries, and the interaction of the mean flow with free surface rheology.

\subsection{Interaction of the viscous mean flow and nearly inviscid Faraday waves in almost circular containers}

Among the various containers considered in Faraday waves studies, circular containers are of particular interest [79], and may lead to very complex dynamics in certain narrow regions in parameter space, as has been demonstrated experimentally $[80,81]$; the mean flow plays a role in this complexity [76]. Near threshold, in contrast, the amplitude of the standing waves produced by the parametric forcing in circular containers decouples from the viscous mean flow. This couples instead only to the spatial phase of the pattern, similarly to what happens in annular containers with strictly circular walls (see the next subsection) and in related circular geometries [82]. As pointed out in [76], however, the situation changes dramatically when the shape of the container is perturbed from circular to ellipsoidal. This is because in this case the mean flow couples to the amplitudes as well as the spatial phases of the resulting pattern. The ellipticity breaks the rotational symmetry of the primary waves and selects two standing oscillations with nodes along either the major or the minor axis of the ellipse. These two standing waves are known as pure modes and denoted by $\mathrm{P}_{ \pm}$; their complex amplitudes are denoted by $X$ and $Y$. In the case of a slightly elliptical container, the analysis can be greatly simplified by treating the ellipticity as a small perturbation. In this limit, the ellipticity can be ignored in the description of the mean flow, which couples to the primary oscillatory flow only through the azimuthal velocity component, denoted by $v$. If, in addition, the mean flow Reynolds number is small, then the description greatly simplifies because the azimuthal velocity component $v$ decouples from the radial and axial components, and furthermore, is given by a linear equation. Therefore, under these assumptions, the weakly nonlinear description of the system is provided by a coupled system of (i) two nonlinear, complex ordinary differential equations that provide $X$ and $Y$ and (ii) a linear partial differential equation, with nonlinear (quadratic) boundary conditions depending on the complex amplitudes $X$ and $Y$. Consequently, this is one of the simplest cases where the viscous mean flow interacts with the surface waves, and also one where the presence of the viscous mean flow has a dramatic effect on the dynamics of the system even near threshold.

For the sake of brevity, this system of coupled amplitude mean flow equations is not reproduced here; see [83] for details. Instead, a bifurcation diagram is shown in Fig. 2-a that provides the response of the 


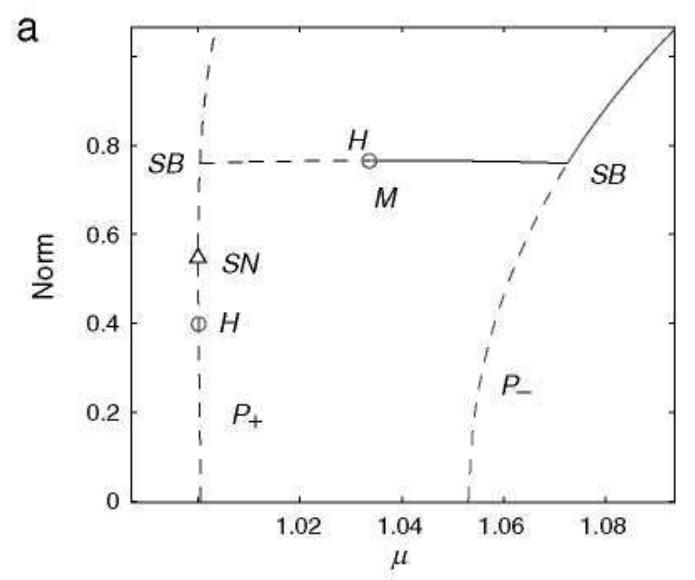

b
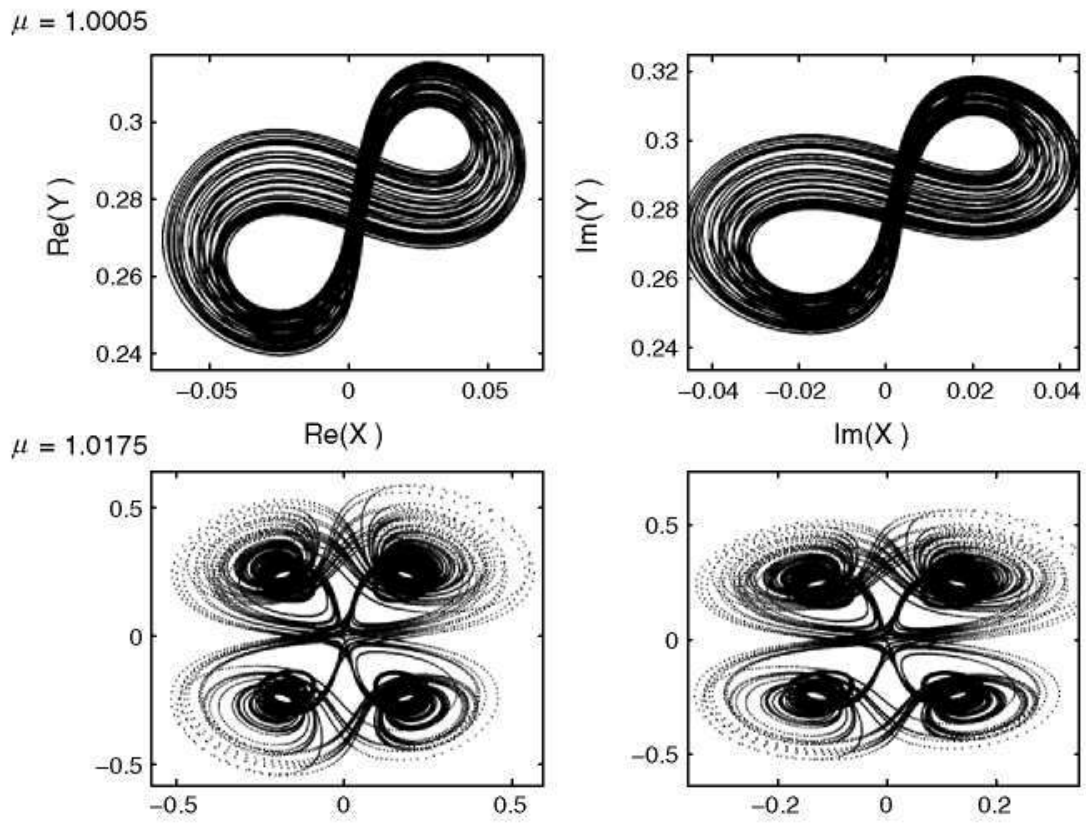

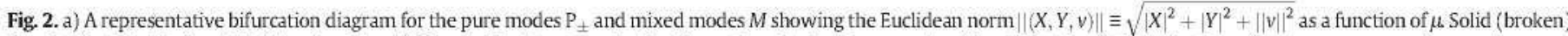
lines indicate stable (unstable) steady states. b) Two stable attractors, plotting the real vs. the imaginary parts of $X$ and $Y$, at the indicated values of $\mu$.

system as the rescaled forcing amplitude $\mu$ increases, with fixed values of the remaining parameters of the system. The steady states of this system are of two types: (i) pure modes corresponding to pure standing waves and associated with vanishing azimuthal viscous mean flow,

$P_{+}:(X, Y, v)=\left(0, Y_{s}, 0\right), \quad P_{-}:(X, Y, v)=\left(X_{s}, 0,0\right)$,

and (ii) mixed modes, which are accompanied by a nonzero azimuthal viscous mean flow,

$M:(X, Y, v)=\left(X_{s}, Y_{s}, v_{s}\right)$.

As shown in Fig. 2, the system possesses three branches of steady states, containing both types of pure modes and mixed modes. In addition to the primary bifurcations that lead to the pure modes, the system exhibits various secondary bifurcations, namely, saddle-node (SN), symmetrybreaking (SB), and Hopf $(\mathrm{H})$ bifurcations [84] showing a complexity that stems entirely from the mean flow. In fact, without the mean flow, only the $\mathrm{P}_{ \pm}$branches would be present, and only that part of the $\mathrm{P}_{+}$ branch above the saddle-node (SN) would be stable; the $\mathrm{P}_{-}$-branch would be entirely unstable. As can be seen in the bifurcation diagram, the mean flow stabilizes a part of the $\mathrm{P}_{-}$branch and destabilizes the upper part of the $P_{+}$branch. Thus, no stable steady state exists for $1<\mu<\mu_{H}^{M}$, (where $\mu_{H}^{M}$ is the forcing amplitude at which the mixed modes undergo a Hopf bifurcation), which implies that the first state observed once $\mu$ exceeds the threshold value $\mu=1$ must be a timedependent state which, as shown in Fig. 2-b, may exhibit very complex dynamics.

In summary, the above discussion illustrates that interaction with the mean flow may produce very complex dynamics even near threshold.

\subsection{Parity breaking of Faraday waves in annular containers}

Let us now consider a vertically vibrated, perfectly circular annular container. For this geometry, it has been experimentally found [85] that the system shows peculiar traveling waves, which are standing in a rotating (at a constant speed) reference frame and are almost sinusoidal in the azimuthal direction. This was striking because these waves exhibit reflection symmetries about the radial planes containing the crests of the wavetrain, which in principle prohibits rotation. Such a paradox can be explained by noting that the viscous mean flow produced by 
the primary waves is fully nonlinear and thus may exhibit spontaneous symmetry-breaking in spite of the fact that the forcing is reflectionsymmetric (because it is produced by the reflection-symmetric primary waves). This nonsymmetric viscous mean flow produces a nonsymmetric state of the system that is compatible with rotation.

A first analysis of the coupled amplitude mean flow equations for this system considering a two-dimensional annulus was performed in [86], where it was shown that in this case the mean flow is coupled with the phase of the waves (namely, the azimuthal position of one of the wave crests), denoted hereafter as $\psi$. It should be noted that because the forcing of the viscous mean flow is quadratic, the forcing boundary conditions are spatially periodic, with a period equal to one half of the period of the primary oscillating waves, $L$. The simplest patterns, denoted hereafter as $\mathrm{SW}(\mathrm{L} / 2)$ are standing waves whose mean flow exhibits a period $L / 2$. The analysis showed that the reflection symmetry of the mean flow is in fact broken and that this promotes drift, yet the primary bifurcation is a Hopf bifurcation of the coupled amplitude mean flow equations. Thus, the resulting states are oscillatory, namely, they rotate back and forth. Steadily traveling waves like the ones visualized in [85] are also present for larger values of the forcing as a consequence of a secondary bifurcation. However, the experiment found these traveling waves at the primary bifurcation. Thus, the analysis seems to confirm the above conjecture that the drift of the standing waves can be due to a symmetry-breaking related to the mean flow, but something is still missing since the sequence of the bifurcations predicted by the theory differs from the experimental observations.

An important point is that the experiments were performed using tap water, while the theory in [86] assumed for simplicity a clean surface. Thus, the effect of free surface contamination (modeled with shear and dilatational viscosity, which are indistinguishable in the two-dimensional annulus where the free surface is one-dimensional) was added to the formulation in [87], where it was seen that contamination dramatically changes the boundary layer attached to the free surface. In fact, highly contaminated free surfaces behave as they were inextensible, which produces a boundary layer similar to that attached to the solid walls. The dynamics of the system show two distinguished regimes, for low and high surface viscosity, but the results in both regimes are qualitatively similar. In both regimes the dynamics can be understood in terms of two nondimensional parameters, the mean flow Reynolds number Re and a parameter $\Gamma$ that measures the relative strength of the lower and upper boundary layers attached to the bottom of the container and the free surface, respectively. As anticipated (see Eq. (20)), Re is proportional to the square of the amplitude of the primary waves and $\Gamma$ is normalized such that $0 \leq \Gamma \leq 1$, with the extreme values $\Gamma=0$ and 1 corresponding to the cases in which forcing comes from the lower and upper boundary layers, respectively.

As in the previous subsection, details are omitted for the sake of brevity (see [87]) and we just describe a representative case. Fig. 3-left shows a typical Re vs. $\Gamma$ diagram locating the primary instability of the basic standing waves, which are stable in the region left of the instability curve. As can be seen, the primary bifurcation is a Hopf bifurcation for small $\Gamma$, which is consistent with the results in [86] for a clean surface $(\Gamma=0)$ and provides a periodic standing wave denoted in Fig. 3 as PSW $(L / 2)$, whose spatial period is $L / 2$. In the opposite limit, as $\Gamma$ approaches 1 , the primary bifurcation is a parity breaking bifurcation [88] that generates the steadily traveling waves encountered experimentally in [85]. These drifting states exhibit a spatial period $\mathrm{L} / 2$ and are denoted by $\operatorname{TW}(\mathrm{L} / 2)$. At intermediate values of $\Gamma$, the primary instability is a spatial period doubling bifurcation that produces standing waves, denoted by $S W(L)$, with a spatial period $L$. Note that the drift velocity ( $=d \psi / d t$, where $\psi$ is the phase of the primary waves) vanishes at the primary instability; beyond the primary instability, as Re increases, this velocity first increases along a first branch of TW $(L / 2)$ and then decreases along a second branch of steadily traveling waves with spatial period $L$, denoted by $T W(L)$; the streamlines of the viscous mean flow in moving axes for two representative points on these two branches are plotted in Fig. 4, where it is evident that the reflection symmetries of the primary oscillating waves are broken in the viscous mean flow. Also note in Fig. 3-right that the system shows more complex chaotic attractors in a narrow window of the second branch.

To summarize, the mean flow may explain propagative phenomena that cannot be understood from a formulation based only on the primary wave dynamics. In larger domains amplitude inhomogeneities can develop and these also couple to the mean flow [78].

\section{A preliminary analysis of compressed-expanded monolayers over a liquid layer}

In the above discussion, we have concentrated on the limit where the vibrating frequency is large compared to the inverse of the viscous timescale. We now consider the opposite limit, taking for illustration a configuration that is relevant to certain experimental devices used to measure surface rheology produced by surfactant monolayers [28]; such systems have received steady attention in the literature [89]. In this configuration, a surfactant monolayer is deposited onto the free surface of a liquid layer of depth $d$ between two parallel horizontal barriers, which are only slightly immersed in the liquid. The barriers are oscillated horizontally in counterphase, with a frequency $\omega$ and a nondimensional amplitude $a$, measured relative to a half of the mean distance between the barriers, $L$. The instantaneous value of the surface tension at a point located symmetrically between the barriers is recorded versus time. If the motion is sufficiently slow, the surfactant concentration is spatially uniform and the surface tension will follow the equilibrium values in a reversible way. Departure from reversibility is usually assumed to be due to nonequilibrium effects $[89,90]$, but it may also be due to irreversible coupling to the fluid dynamics in the
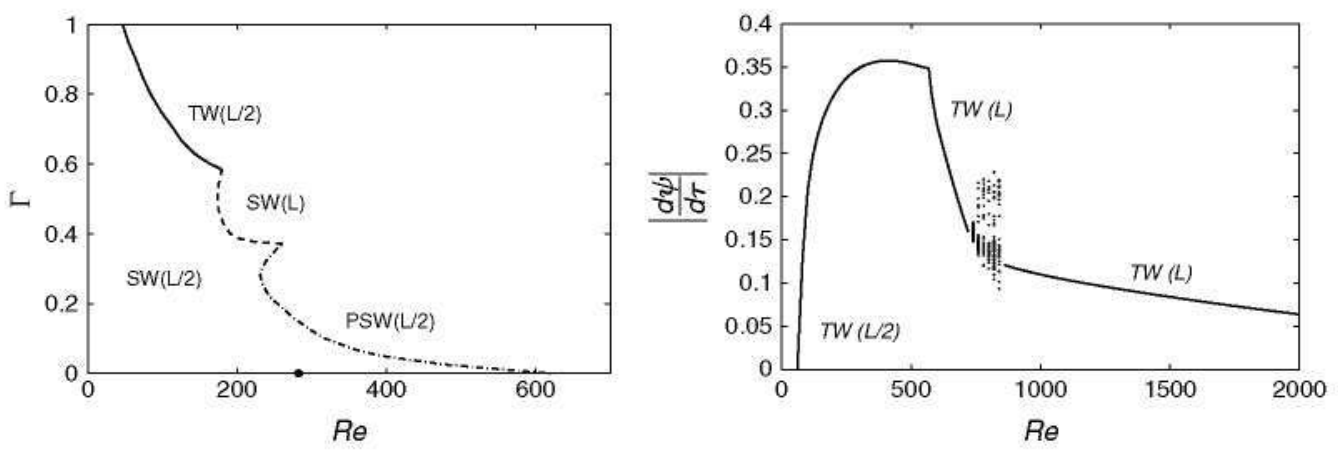

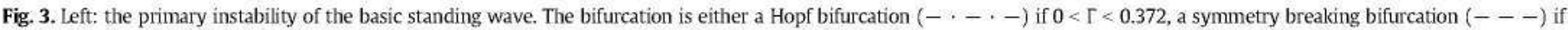

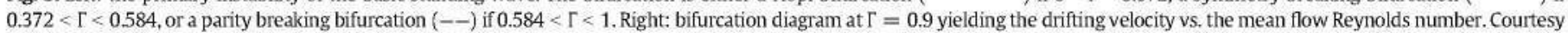
of Elena Martin. 

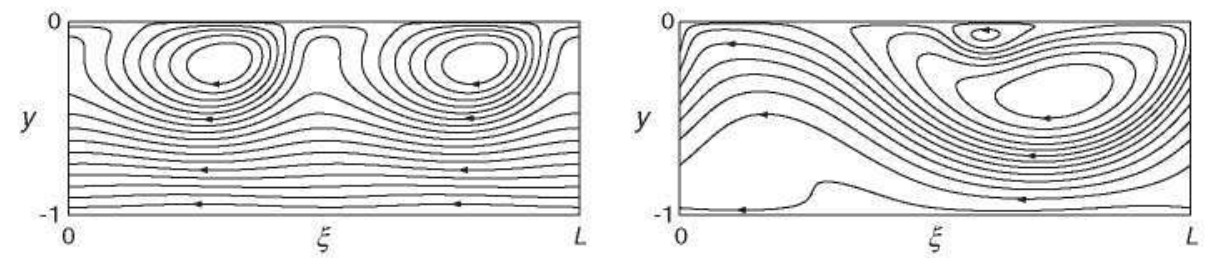

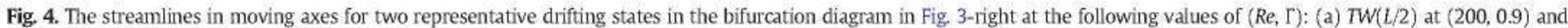
(b) $T W(L)$ at $(600,0.9)$. Courtesy of Elena Martin.

bulk phase if the motion is not sufficiently slow, as we show now. Reversibility is appreciated by plotting the so-called Lissajous orbits, which are plots of the surface pressure П (namely, the surface tension drop from its clean value due to the presence of surfactant) vs. the area of the monolayer $A$. For reversible dynamics, these orbits move back and forth on the curve $\Pi=\Pi\left(A_{0} / A\right)$, where $A_{0}$ is the initial value of the area. For irreversible dynamics, in contrast, Lissajous orbits are open curves. To simplify the analysis, we consider a twodimensional formulation, assume an insoluble surfactant, and neglect the effects of surface viscosity, surfactant diffusion, the free surface deformation, and the menisci near the barriers. The additional assumptions $L \gg d$ and $\omega \ll \nu / d^{2}$, and $\rho$ g d $\gg \Pi$, where $\rho$ and $\nu$ are the density and the kinematic viscosity in the bulk phase, and g is the gravitational acceleration, allow for a lubrication approximation that produces the following evolution equation for the surfactant concentration c,

$(\rho \nu / d) \partial_{t} c=\partial_{x}\left[c \partial_{x} \Pi(c)\right]$ in $-L(1+a \sin \omega t)<x<L(1+a \sin \omega t)$.

This equation has already been derived and repeatedly used in the context of dynamics of (insoluble) surfactant over thin liquid layers (see [91-93] and references therein). In this limit, the fluid dynamics is slaved to the surfactant concentration gradient and the nonlinear diffusion term in the equation results from the interplay of Marangoni stress and the restoring effect of the fluid dynamics in the bulk.

Eq. (23) applies between the solid barriers. The appropriate boundary conditions at the barriers result from imposing that the velocity of the surfactant monolayer at the free surface coincides with the velocity of the barriers, which in the present approximation leads to

$\partial_{x} \Pi(c)=\mp(\rho v / d) \omega a L \cos \omega t \quad$ at $x= \pm L(1+a \sin \omega t)$.

This boundary condition is compatible with surfactant conservation, as seen integrating Eq. (23) over $-L(1+$ asin $\omega t)<x<L(1+$ asin $\omega t)$, applying integration by parts on the right hand side of the resulting equation, and substituting into Eq. (24), to give

$\frac{d}{d t} \int_{-L(1+a \sin \omega t)}^{L(1+a \sin \omega t)} c d x=0$.

Now, a typical experimental plot for the dependence of surface pressure on the surfactant concentration is provided in Fig. 5-top. Three regimes are visible in this plot, for increasing surfactant concentration, with nonequilibrium effects expected mainly in the concentrated regime. To illustrate how the fluid dynamics promotes irreversibility, Eqs. (23)-(24) are numerically integrated with a uniform initial condition, $c=c_{0}$ at $t=0$ for the case described in the caption of Fig. 5 , which can be considered as representative of typical experimental runs. The results of such integration are provided in the inset of Fig. 5-top and in the bottom plots in this figure. In particular, note that the Lissajous orbit is an open curve, which demonstrates irreversibility. Of course, for still slower forcing frequencies, as $\omega \ll \rho \nu L^{2} /(\Pi d)$, Eq. (19) implies that $c$ is spatially uniform and Eq. (24) readily implies that $c$ is proportional to the inverse of the distance between the barriers, which in turn means that the dynamics is reversible. But this is not the case when the dynamics are not sufficiently slow, as shown above.

In summary, even in the simplest formulation, the fluid dynamics alone are sufficient to provide irreversibility.

\section{Concluding remarks}

Some basic properties of the vibration driven dynamics of nearly inviscid confined liquid systems with interfaces have been reviewed. This is an important issue in many scientific and engineering fields that involve confined fluids due to the presence of external perturbations, noise, or other environmental oscillations that cannot be avoided. It is obviously important to understand the extent to which such vibrations will affect the system of interest. At the same time, many experiments make use of applied vibrations in order to study a particular phenomenon (Faraday waves or cross-waves, for example) or to measure a particular physical property (surface tension, Marangoni elasticity, surface viscosities). In these cases it is important to understand all the effects of vibrations, including the generation of mean flows and the influence of symmetries and resonances. An analysis which fails to account for the more subtle and surprising effects of the fluid mechanics of oscillating flows can easily lead to paradoxes (the presence of 'forbidden' drifting waves, for example) or misleading conclusions (for instance, attributing irreversibility solely to nonequilibrium effects).
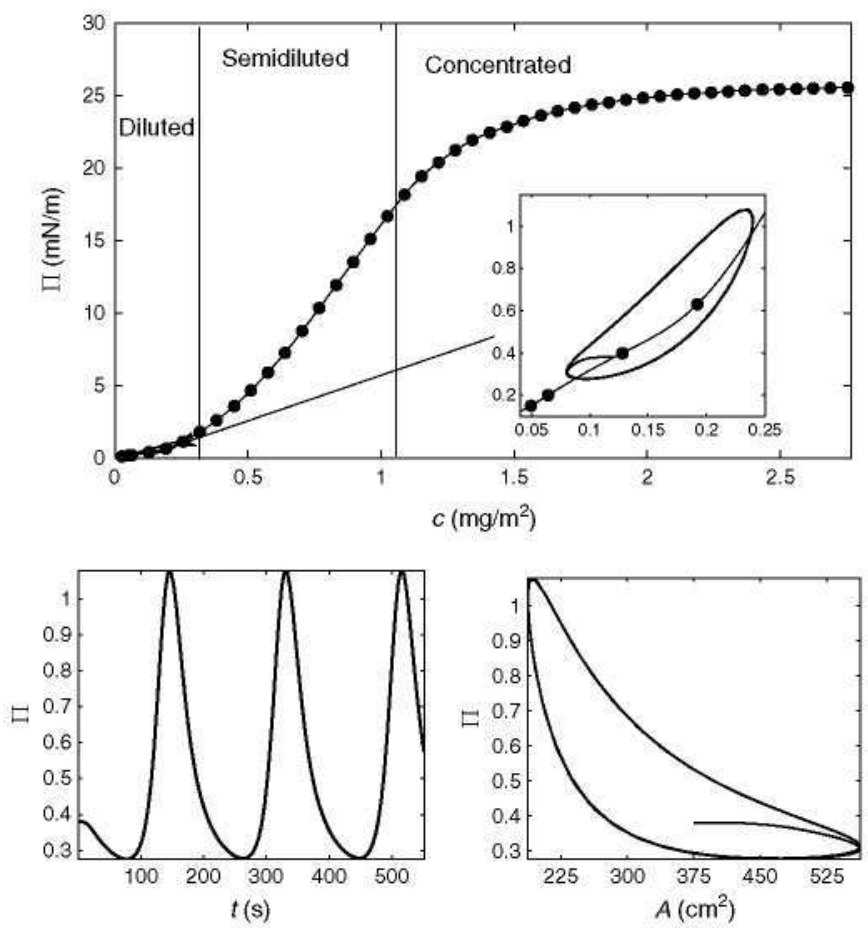

Fig. 5. Top: surface pressure vs. gradient plot for PVAc, as provided in [28]; the inset provides the surfactant concentration at the midpoint between the barriers as resulting from integrating Eqs. (23)-(24) with $\omega=5.4 \mathrm{mHz}, c_{0}=0.12 \mathrm{mg} / \mathrm{m}^{2}, a=0.3$, and $L=15 \mathrm{~cm}$. Bottom: ПIvs, $t$ (left) and II vs. $A$ plots for the case considered in the top plots. 
We presented the basic equations describing vibrated fluids and discussed various kinds of solutions found in them. The forcing was assumed for simplicity to be monochromatic and a distinction was made between fast and slow oscillations, considered with respect to the viscous timescale. The forcing in these systems can be either direct, which produces an immediate response, or parametric, which requires a critical threshold to be surpassed before an instability is observed. The parametric forcing mechanism is dominant in vertically vibrated fluids, while direct forcing is characteristic of horizontally (or obliquely) vibrated fluids. Symmetries are important in determining (in advance of any modeling or computation) the properties of the solutions excited by these different forcing mechanisms.

Viscous mean flows are an important, but often neglected, product of oscillatory forcing. They occur even in the limit of inviscid flow, a surprising property that illustrates that viscosity, however small, has deeper effects on the fluid flow than just damping. The viscous mean flow should not be neglected in fluid flows with interfaces because the corresponding terms in the evolution equations are comparable in size to the usual cubic nonlinearities included in weakly nonlinear analyses. Mean flows, despite their much slower timescale, can couple with the faster oscillatory flow and significantly influence dynamics, generating new bifurcations (instabilities), for example, or inducing symmetrybreaking that can lead to drifting waves.

An interesting new application to surface rheology experiments was considered in Section 5. These are low frequency experiments where fluid dynamics is commonly ignored. In fact, as we show here, it is dangerous to neglect the fluid dynamics generated by oscillatory forcing. Fluid dynamics, even if not apparent via large scale motion or surface waves, can have important and surprising consequences. In this case fluid dynamics is sufficient to explain the irreversible character evident in plots of surface pressure versus surface area. Additional nonequilibrium assumptions are seemingly relevant too, but these must be considered in addition to (not in place of) the fluid dynamics in the bulk phase.

In summary, there are numerous applications involving fluids with free interfaces where vibrations, either environmental or intentionally applied, are important considerations. In these applications it is crucial to understand the basic fluid dynamics that can affect the behavior of the system, including the influence of the relevant forcing mechanism, viscous mean flows, and spatial and temporal symmetries. This review is intended to indicate the necessary first steps in doing so.

\section{Acknowledgments}

This research was partially supported by the Spanish Ministry of Science and Innovation, under Grants TRA2010-18054 (MH and JMV), MTM-2010-21135-C02-02 (FV), and AYA2010-19081 (JP).

\section{References}

[1] McPherson K, Hrovat K. Technical Report NASA TM 2000-209677. NASA Lewis Research Center; 2000.

[2] Nelson E. Technical Report NASA TM 103775. NASA Lewis Research Center; 1994.

[3] Faraday M. Phil Trans R Soc Lond 1831:121:299-340.

[4] Fauve S. In: Martínez Mekler G, Seligman TH, editors. Dynamics of nonlinear and disordered systems. World Scientific; 1995 . p. 67-115.

[5] Edwards WS, Fauve S.J Fluid Mech 1994:278:123-48.

[6] Kudrolli A, Gollub JP. Phys D 1997;97:133-54.

[7] Kudrolli A, Pier B, Gollub JP. Phys D 1998;123:99-111

[8] Arbell H, Fineberg I. Phys Rev E 2002;65:036224.

[9] Benjamin TB, Ursell F. Proc R Soc Lond A 1954:225:505-15.

[10] Wernet A, Wagner C, Papathanassiou D, Müller HW, Knorr K. Phys Rev E 2001;63: 036305 .

[11] Miles J. Henderson D. Annu Rev Fluid Mech 1990:22:143-65.

[12] Barnard BIS, Pritchard WG. J Fluid Mech 1972:55:245-55.

[13] Underhill WB, Lichter S, Bernoff AJ. J Fluid Mech 1991;225:371-94.

[14] Garrett CIR.] Fluid Mech 1970;41:837-49.

[15] Porter J, Tinao I, Laveron-Simavilla A, Lopez CA. Fluid Dyn Res 2012:44:065501.

[16] Perez-Gracia JM, Porter J, Varas F, Vega JM. J Fluid Mech 2013 [in press].
[17] Miles JW. J Fluid Mech 1984:149:15-31.

[18] Funakoshi M, Inoue S. J Fluid Mech 1988;192:219-47.

[19] Feng ZC. Phys Rev Lett 1997;79:415-8.

[20] Hill DF. Phys Fluids 2003:15:1576-87.

[21] Faltinsen OM, Rognebakke OF, Timokha AN. Phys Fluids 2006:18:012103.

[22] Hermann M, Timokha A. Math Mod Meth Appl Sci 2008;18:1845-67.

[23] Faltinsen OM, Timokha AN. Sloshing. Cambridge Univ. Press; 2009.

[24] Wu CH, Chen BF. Ocean Eng 2009;36:500-10.

[25] Vega JM, Knobloch E, Martel C, Phys D 2001;154:313-36.

[26] Knobloch E, Vega JM. Geometry, mechanics and mechanics and dynamics: volume in honor of the 60th birthday of J.E. Marsden. Springer Verlag: 2002 181-222.

[27] Thiele U, Vega JM, Knobloch E.] Fluid Mech 2006:546:61-87.

[28] Hilles H, Monroy F, Bonales LJ, Ortega F, Rubio RG. Adv Colloid Interf Sci 2006;122:67-77.

[29] Herrada MA, Montanero JM, Vega JM. Phys Fluids 2011;23:082102.

[30] James A], Smith MK, Glezer A. J Fluid Mech 2003;476:29-62.

[31] Vukasinovic B, Smith MK, Glezer A.J Fluid Mech 2007;587:395-423.

[32] Noblin X, Buguin A, Brochard-Wydart B. Eur Phys J E 2004;14:395-404

[33] Moisy F, Michon G], Rabaud M, Sultan E. Phys Fluids 2012;24:022110.

[34] Snoeijer JH, Andreotti B. Annu Rev Fluid Mech 2013;45:269-92.

[35] Mathieu E. J Math 1868:13:137-203.

[36] Landau LD, Lifshitz EM. Mechanics. Pergamon Press; 1976.

[37] Stephenson A. Mem Proc Manch Lit Phil Soc 1908;52:1-10.

[38] Schlichting H. Phys Z 1932;33:327-35.

[39] Schlichting H. Boundary-layer theory. 7 th ed. McGraw-Hill; 1979 .

[40] Zhang W, Viñals J. J Fluid Mech 1997:336:301-30.

[41] Martel C, Nicolas JA, Vega JM.J Jluid Mech 1998:360:213-28 [See also Corrigendum, 373 (1998) 379].

[42] Epstein T, Fineberg J. Phys Rev E 2006;73:055302.

[43] Porter J, Silber M. Phys Rev Lett 2002:89:084501.

[44] Topaz CM, Porter J, Silber M. Phys Rev E 2004;73:066206.

[45] Sandstede B, Scheel A. Nonlinearity 1995;8:333-65.

[46] Moehlis J, Knobloch E. Phys Rev Lett 1998;80:5329-32.

[47] Wolf GH. Z Physik 1969;227:291-300.

[48] Wolf GH. Phys Rev Lett 1970;24:444-6.

[49] Lapuerta V, Mancebo F]. Vega JM. Phys Rev E 2001;64:016318.

[50] Varas F, Vega JM. J Fluid Mech 2007;579:271-304.

[51] Lord Rayleigh JWS. Phil Trans R Soc Lond 1883;175:1-21.

[52] Lord Rayleigh JWS. The theory of sound, Vol. I, Dover; 1945.

[53] Taylor S. Proc R Soc Lond 1878:27:71-6.

[54] Afenchenko VO, Ezersky AB, Kiyashko SV, Rabinovich MI, Weidman PD. Phys Fluids 1998:10:390-9.

[55] Vega JM, Higuera FJ, Weidman PD.J Fluid Mech 1998;372:213-30.

[56] Longuet-Higgins MS. Phil Trans R Soc A 1953;245:535-81.

[57] Riley N. Annu Rev Fluid Mech 2001;33:43-65.

[58] Padmanabhan N, Pedley T]. J Fluid Mech 1987;178:325-43.

[59] Lighthill J.J Fluid Mech 1992;239:551-606

[60] Riley N.] Fluid Mech 1992;242:387-94.

[61] Yan B, Ingham DB, Morton BR. J Fluid Mech 1993;252:147-71.

[62] Phillips OM. The dynamics of the upper ocean. Cambridge Univ. Press; 1977.

[63] Liu AK, Davis SH. J Fluid Mech 1977;81:63-84.

[64] Craik ADD. J Fluid Mech 1982;116:187-205.

[65] Craik ADD. Wave Interactions and Fluid Flows. Cambridge Univ. Press; 1985.

[66] Iskandarani M, Liu PL.F.J Fluid Mech 1991:231:417-37.

[67] Craik ADD, Leibovich S. J Fluid Mech 1976:73:401-26.

[68] Leibovich S. Annu Rev Fluid Mech 1983;15:391-427.

[69] Mollot D], Tsamopoulos J, Chen TY, Ashgriz A.J Fluid Mech 1993:255:411-35.

[70] Nicolas JA, Vega JM. J Fluid Mech 1996;328:95-128.

[71] Anilkumar AV, Grugel RN, Shen XF, lee CP, Wang TG. J Appl Phys 1993;73:4165-70.

[72] Nicolas JA, Rivas D, Vega JM. Z Angew Math Phys 1997;48:389-423.

[73] Nicolas JA, Rivas D, Vega JM. J Fluid Mech 1998;354:147-74.

[74] Jurish M, Löser W. J Cryst Growth 1990:102:214-22.

[75] Kuhlmann HC. Thermocapillary convection in models of crystal growth. SpringerVerlag; 1999.

[76] Higuera M, Vega JM, Knobloch E.J Nonlinear Sci 2002:12:505-51.

[77] Vega JM, Rüdiger S, Viñals J. Phys Rev E 2004;70:0406306.

[78] Lapuerta V, Martel C, Vega JM. Phys D 2002;173:178-203.

[79] Miles JW. J Fluid Mech 1984;149:1-14

[80] Ciliberto S, Gollub JP. Phys Rev Lett 1984;52:922-5.

[81] Ciliberto S, Gollub JP. J Fluid Mech 1985;158:381-98.

[82] Higuera M, Nicolas JA, Vega JM. Phys Fluids 2002;14:3251-71.

[83] Higuera M, Vega JM, Knobloch E. Phys D 2005;201:83-120.

[84] Guckenheimer J, Holmes P. Nonlinear oscillations dynamical systems and bifurcation of vector fields. Springer-Verlag; 1983.

[85] Douady S, Fauve S, Thual O. Europhys Lett 1989;10:309-15.

[86] Martin E, Martel C, Vega JM. J Fluid Mech 2002;467:57-79.

[87] Martin E, Vega JM. J Fluid Mech 2006;546:203-25.

[88] Crawford JD, Knobloch E. Annu Rev Fluid Mech 1991;23:341-87

[89] Saguis LMC. Rev Mod Phys 2011:84:1367-403.

[90] Fainerman VB, Lylyk SV, Aksenenko EV, Petkov JT, Yorke J, Miller R. Colloid Surf A 2010;354:8-15.

[91] Renardy M. Nonlinear Anal 1996;26:1207-19.

[92] Matar OK, Troian SM. Chaos 1999:9:141-53.

[93] Dassaud AD, Matar OK, Troian SM. J Fluid Mech 2005;544:23-51. 